\title{
DUST PROPERTIES OF TWO NEW CAVITY STRUCTURES NEARBY ASYMPTOTIC GIANT BRANCH STARS: THE IRAS SURVEY
}

\author{
Sujan Prasad Gautam ${ }^{1}$, Ashok Silwal ${ }^{2}$, Mijas Tiwari ${ }^{3}$, Seema Subedi² ${ }^{2}$ Manish Khanal ${ }^{4}$, Ajay Kumar Jha ${ }^{*}$ \\ ${ }^{1}$ Central Department of Physics, Tribhuvan University, Kirtipur, Nepal \\ ${ }^{2}$ Patan Multiple Campus, Tribhuvan University, Lalitpur, Nepal \\ ${ }^{3}$ Department of Physics and Astrophysics, University of Delhi, Delhi, India \\ ${ }^{4}$ Amrit Campus, Tribhuvan University, Kathmandu, Nepal \\ *Corresponding author: astroajay123@gmail.com
}

(Received: July 11, 2021; Revised: December 8, 2021; Accepted: December 21, 2021)

\begin{abstract}
We studied the dust properties of two cavity structures (namely FIC21+54 and FIC16-56) nearby Asymptotic Giant Branch (AGB) stars using Infrared Astronomical Satellite (IRAS) maps. Dust color temperature, Planck function, dust mass, and visual extinction with their distribution within the region of interest were examined. The temperature of dust was found to lie in the range of $22.24 \pm 0.81 \mathrm{~K}$ to $23.27 \pm 0.21 \mathrm{~K}$, and $25.12 \pm 0.43 \mathrm{~K}$ to $26.17 \pm 0.62 \mathrm{~K}$, and the mass of dust was obtained within the range of $4.21 \times 10^{26} \mathrm{~kg}$ to $3.6 \times 10^{27} \mathrm{~kg}$, and $2.1 \times 10^{27} \mathrm{~kg}$ to $3.31 \times 10^{28} \mathrm{~kg}$, for FIC21+54 and FIC16-56, respectively. Some unusual behaviors on the distribution of dust temperature indicated the effect of nearby sources within the studied structures. Moreover, we observed the trend of dust particles along the major and minor diameters, and plots represented that the particles were oscillating with a sinusoidal pattern in both cavities. The negative slope between $25 \mu \mathrm{m}$ and $60 \mu \mathrm{m}$ in far-infrared spectral distribution was encountered for both structures, which portrayed less number density of particles in $60 \mu \mathrm{m}$ band; interaction between AGB wind and the ambient interstellar medium could be the possible reason behind this. These findings support the prior results of cavity structures nearby AGB stars within the galactic plane $-10^{\circ}<\mathrm{b}<+10^{\circ}$.
\end{abstract}

Keywords: External factor - AGB wind, extinction, ISM - dust, Planck function, stars - AGB

\section{INTRODUCTION}

Last evolutionary stage of the low and intermediate-mass $(0.8 \mathrm{M} \odot<\mathrm{M}<10 \mathrm{M} \odot)$ star devoid of nuclear fuel is known as the Asymptotic Giant Branch (AGB) star (Herwig, 2005). In its core region, as the star consumes the supply of hydrogen, the core compresses, which results in the increment in its temperature and causes the extension of the boundary layer; ultimately, it cools the star, resulting in their shift to a red giant, upper-right corner of the HR diagram (Iben Jr, 1967). At around $3 \times 10^{8} \mathrm{~K}$, helium burning begins in the core, which prohibits further cooling and increases their luminosity; this phenomenon is common to stars with a mass of more than about $2 \mathrm{M} \odot$ (Vassiliadis \& Wood, 1993). The star goes to the right and upward portion of the HR diagram when helium fusion is completed in the center, which is known as the AGB phase.

AGBs are regarded as the major dust sources to the galaxies through their strong wind, which recreates the surroundings with dust particles (Gehrz, 1989). The red giants form carbon $(\mathrm{C})$ or oxygen $(\mathrm{O})$ rich solid dust in the stellar photosphere depending upon the number of dredgeup events and its evolutionary stages. In the spectral energy distribution (SED) of AGB stars, prominent dust characteristics can be analyzed, including 9.7 and $18 \mu \mathrm{m}$ silicates features in O-rich $\mathrm{AGBs}$, and $11.3 \mu \mathrm{m}$ silicon carbonate dust features in C-rich stars (Gobrecht et al., 2016). By utilizing interferometric data, Danchi et al. (1994) observed that the dust forming area is situated within $10 \mathrm{R}^{*}$, where $\mathrm{R}^{*}$ represents the star's radius from the stellar photosphere. The stellar pulsation produces the periodic shock, which pushes warm and dense gas layers to the large radii, making a favorable environment for the formation of solids. Due to radiation pressure on dust particles and the high opacity of dust, this event helps to accelerate the stellar wind (Bowen, 1988). These thick layers facilitate the production of molecules and various gas-phase components in both C \& O-rich AGBs (Cernicharo et al., 2008; Justtanont et al., 2012). The non-equilibrium phenomena driven by the periodic flow of the shocks regulate the creation of $\mathrm{O}$ or $\mathrm{C}$-bearing components in the wind of both carbon- and oxygen-rich AGBs (Gobrecht $e t$ al., 2016). The $\mathrm{C} / \mathrm{O}$ ratio and partial dissociation of the $\mathrm{CO}$ molecule both impact the growth of molecules within the photosphere. In a carbon-rich star, the shock wave dissociated oxygen atoms that form O-bearing components such as $\mathrm{H}_{2} \mathrm{O}$ in the wind, but in oxygen-rich $\mathrm{AGB}$, carbons are released due to shock, which forms C-bearing species (Willacy \& Cherchneff, 1998; Groom, 2018). Several literatures (e.g.: Neufeld et al., 2011; Justtanont et al., 2012) provide a basic for the existence of warm water, $\mathrm{HCN}$ in the C-rich AGBs. These shreds of evidence support the shock-induced mechanism in the creation of molecules in the wind of AGBs. There is almost (90-98) \% mass loss of AGB stars in the form of massive winds (Rees, 1984) in the interstellar medium (ISM). 
Dust in ISM was first observed through interstellar extinction curves. It absorbs the Ultraviolet (UV) radiation and re-emits infrared radiation, suggesting that $A G B$ is a major IR source; this UV radiation causes the molecular dissociation of dust, providing the site for a star formation (Gehrz, 1981; Gobrecht et al., 2016). The study of different types of AGB stars helps to understand the nature of the mass loss to ISM (Vassiliadis \& Wood, 1993; Ventura et al., 2012). The O-rich AGB mainly produces silicates-type grains in the outflow, $\mathrm{C}$-rich produces mainly carbonaceous grains in the outflow (Gautam \& Aryal, 2020). Due to the different dust compositions of these two types of AGB stars, we obtain different infrared spectral features. Suh and Kwon (2009) prepared a detailed catalogue of AGB stars in the IRAS point source catalogue. From the study of farinfrared (FIR) loops, Kiss et al. (2004) and Könyves et al. (2007) stated that such structures were probably generated by high-pressure events, such as AGB wind, in the past. Several studies (e.g., Aryal \& Weinberger, 2006, Aryal et al., 2010) presented the discovery of new nebula structures using the IRAS survey. In the past, various studies (e.g., Jha \& Aryal, 2018; Gautam \& Aryal, 2019a; Gautam \& Aryal, 2019b; Sapkota \& Aryal, 2019; Gautam \& Aryal 2020; Tiwari et al., 2021) aimed to investigate dust properties of far-infrared cavities located around the AGB stars at different coordinates using IRAS maps.

The study of dust properties in the ISM serves essential reference for understanding dust status around the various sources, and ultimately, it helps to upgrade our idea of shaping mechanisms in the ISM. This paper presents results from the study of the physical parameters, namely temperature and mass of the dust, visual extinction, radiation intensity distribution, and far-infrared spectral distribution of two new far-infrared cavity structures. We compared our findings with the previously studied structures to validate the existing results for the new cavities within a galactic plane $-10^{\circ}<\mathrm{b}<+10^{\circ}$.

\section{Far-Infrared cavities}

Using the Sky View Virtual Observatory (http://skyview.gsfc.nasa.gov/), we performed a methodical investigation at 60 and 100-micron IRAS survey to find a new cavity structure. We investigated far-infrared cavities around the 1168 carbon-rich AGB stars presented by Suh and Kwon (2009) in their catalogue. We selected the far-infrared cavity structures around the AGB stars based on the following similar selection criteria as performed by Gautam and Aryal (2019a): (i) At $100 \mu \mathrm{m}$ IRAS maps, the central region of the structure should have the lowest flux, (ii) the major width of the structure should be greater than $0.3^{\circ}$ (iii) it should maintain the position within the $3^{\circ}$ area from C-rich AGB stars, and (iv) it should be situated within the Galactic coordinates $-10^{\circ}<\mathrm{b}<10^{\circ}$. For our study, we selected two new far-infrared cavity structures. Minimum flux zones were defined by selecting the region with depression in the far-infrared background emission by photometric analysis using SalsaJ software. The first structure, namely FIC21+55 (size 61.8 pc $\times 46.5$ pc), was situated in the sky coordinates at R.A. (J2000): $21 \mathrm{~h} 32 \mathrm{~m}$ 44.47s, and Dec. (J2000): +55d 15m 16.8s around C-rich AGB star, AGB21+54, located at R.A.: $21 \mathrm{~h} 28 \mathrm{~m} \mathrm{58.56s,}$ and Dec.: $+54 \mathrm{~d} 33 \mathrm{~m} \mathrm{34.57s}$, about $\sim 3580$ pc distance away (calculated using parallax value given by Gaia DR2, https://www.cosmos.esa.int/web/gaia/dr2). Similarly, second structure FIC16-56 (size $25.55 \mathrm{pc} \times 17.92 \mathrm{pc}$ ) was situated at R.A. (J2000): 16h 37m 18.12s and Dec. (J2000): -56d 35m 25.3s nearby C-rich AGB star - AGB16-56

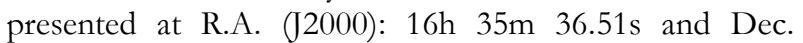
(J2000): -56d 17m 31.8s, about $\sim 2449$ pc away from us.

\section{MATERIALS AND METHODS}

At 60 and $100 \mu \mathrm{m}$, we retrieved the FITS (Flexible Image Transport System) images of both selected structures. We used the tool ALADIN v2.5 (https://aladin.u-strasbg.fr/) to analyze those images. We constructed contours to separate the minimal flux zones. Further, the appropriate flux at 60 and 100 microns at each pixel within the area of interest was measured. The SIMBAD database (https://simbad.u-strasbg.fr/simbad/) was used to investigate the possible contributing sources for the background flux. Background flux within the cavities was estimated using the SalsaJ software. Finally, we subtracted the background flux from 60- and 100-micron flux density measured for both structures. Methods of calculating the temperature of the dust, Planck function, the dust mass, structure size, and visual extinction of the cavity structures around the AGBs are briefly discussed below.

\section{Dust color temperature}

The mathematical formula expressed by Wood et al. (1994), later revised by Arce and Goodman (1999), Dupac et al. (2003), and Schnee et al. (2005), was used for the estimation of the dust temperature at each pixel. According to them, the formula to estimate the temperature of dust $T_{d}$ is given by,

$T_{d}=\frac{-96}{\ln \left\{R \times 0.6^{(3+\beta)}\right\}}, R=\frac{F(60 \mu m)}{F(100 \mu m)}$

Where $F(60 \mu m)$ and $F(100 \mu m)$ represent the flux densities at 60 and 100 microns, respectively, the value of $\beta$ is known as the spectral emissivity index, and it is determined by grain properties including composition, size, compactness, etc. (Jha et al., 2017; Jha and Aryal, 2018). A pure blackbody has a value of $\beta=0$, the amorphous layerlattice matter has $\beta \sim 1$, and the metals and crystalline dielectrics have $\beta \sim 2$ (Gautam \& Aryal, 2020). We used $\beta=2$ for our calculation; Equation (1) provides the temperature of dust from 60- and 100-microns bands of IRAS.

\section{Planck function}

The Planck function or radiation intensity depends on the wavelength (frequency) and temperature. It can be 
estimated using the expression of Beichman et al. (1998). The calculated Planck function at each pixel was used for the evaluation of dust mass.

$B(v, T)=\frac{2 h c}{\lambda^{3}}\left(\frac{1}{e^{\frac{h c}{\lambda k T}-1}}\right)$

where $b$ represents the Planck constant, $c$ is the speed of light in a vacuum, and $v$ indicates the frequency at emission, and $T$ is the calculated dust temperature at each pixel. Equation (2) implies that $B(v, T)$ at larger wavelengths gives a greater value than that of smaller wavelengths. For the fixed temperature, if the wavelength of the image increases, the $B(v, T)$ range becomes narrow.

\section{Dust mass}

To compute the dust mass, at first, we measured the flux at 100-micron IRAS maps and applied Hildebrand's formula (Hildebrand et al., 1977; Hildebrand, 1983), which depends on various properties of dust particles, dust temperature, and distance. The expression for the estimation of dust mass is given by,

$M_{d u s t}=\frac{4 a \rho}{3 Q(v)} \frac{F(v) D^{2}}{B(v, T)}$

Where $a, \varrho$, and $Q(v)$ are the size, density, and emissivity of the dust particles, respectively. $F(v)=f \times 5.228 \times$ $10^{-9} \times$ MJysr $^{-1}$, represents the flux density of the region of study. $\mathrm{B}(\mathrm{U}, \mathrm{T})$ is the Planck function calculated using Equation (2). By applying the constant values, $a=0.1 \mu \mathrm{m}$, $\varrho=3000 \mathrm{kgm}^{-3}$, and $Q(v)=0.0010$ for $100 \mu \mathrm{m}$ and 0.0046 for $60 \mu \mathrm{m}$, respectively as suggested by Young et al. (1993), the Equation 3 reduces to the form,

$M_{\text {dust }}=0.4\left[\frac{F(v) D^{2}}{B(v, T)}\right]$

Equation (4) was used for the calculation of the dust mass within the selected cavity structures.

\section{Visual extinction}

Visual extinction can be obtained by using the technique of Wood et al. (1994). According to them, the expression for the calculation of visual extinction is given by,

$$
\begin{aligned}
& A_{v}(\mathrm{mag})=15.078\left[1-\exp \left(-\frac{\tau_{100}}{641.3}\right)\right] \\
& \text { Where, } \tau_{100}=\frac{F_{\lambda}(100 \mu m)}{B_{\lambda}\left(100 \mu m, T_{d}\right)}
\end{aligned}
$$

Here, $\tau_{100}$ is called optical depth at $100 \mu \mathrm{m}$ wavelength. $F_{\lambda}$ and $B_{\lambda}$ are the flux density and Planck function respectively at the band of 100-micron.

\section{Size of the structure}

To measure the major and minor diameter of the structure, we adopted a similar method used by Gautam and Aryal (2019a). The expression for the calculation of the size of the structure is given by,

$L=D^{\prime} \times \theta$

Here $D^{\prime}$ is the distance to the structure from us, and $\theta$ is the pixel size (in radian).

\section{RESULTS AND DISCUSSION}

This section discusses the physical properties of two new far-infrared cavity structures, namely FIC21+55 and FIC16-56 located within the galactic plane $-10^{\circ}<\mathrm{b}<+10^{\circ}$ and compares the results with the previous studies.

Figures 1(a) and 2(a) show the JPEG image of the cavity structures FIC21+55 and FIC16-56, respectively, where the central position of the cavity is shown by ' + ' sign. Black colored area indicates the lower flux region, and the yellowcolored area depicts the maximum flux-region. The study region was separated by the contour, and the flux densities at 60 microns and 100 microns were computed using ALADIN v2.5 software. The background fluxes contributed by other nearby sources (stars, IRAS sources, etc.) were subtracted, for the accurate study of the distribution of flux of the cavity structure, using SalsaJ software (http://euhou.net/index.php/salsaj-softwaremainmenu-9). The contour plot of the flux density at $100-$ micron IRAS map for both cavities was drawn using the software ORIGIN v8.5, which are represented in Figures 1(b) and 2(b). The colored region in the figure represents the flux density within the right ascension (R.A.) and declination (Dec.) associated with the structures. The fluxregion in FIC21+55 was found to be separated into the region with different pieces with minimum flux-region at the center and maximum flux-region across the boundary; however, distribution for FIC16-56 was separated into different layers with minimum flux-region covered by maximum flux regions. Similar nature of the flux distribution was also encountered and studied in the previous cavity structures (Gautam \& Aryal, 2019a, b; Gautam \& Aryal, 2020, Tiwari et al., 2021).

Figures 1(c) and 2(c) show the linear fit between flux density at 100 -micron $F(100)$ and 60 -micron $F(60)$ of the cavity structures FIC21+55 and FIC16-56, respectively. The slope of the line was 0.20 and 0.30 , and they were positively correlated with the correlation coefficient, $\mathrm{R}=$ 0.84 and 0.93, for FIC21+55 and FIC16-56, respectively. These obtained values of the slope of the best fit were used to set the error bars as suggested by Weinberger and Armsdorfer (2004), Jha and Aryal (2017), and Gautam and Aryal (2019a). Figures 1(d) and 2(d) show the contour map of the dust color temperature of each pixel of the cavities FIC21+55 and FIC16-56, respectively. We evaluated the dust color temperature of each pixel of the cavities using Equation (1) and found it in the range of $22.24 \pm 0.81 \mathrm{~K}$ to $23.27 \pm 0.21 \mathrm{~K}$ for FIC21+55, and $25.12 \pm 0.43 \mathrm{~K}$ to 26.17 $\pm 0.62 \mathrm{~K}$ for FIC16-56, with an offset of about $1 \mathrm{~K}$ for both structures. This small value of offset signified that the dust in the cavities has a higher possibility to be in thermal equilibrium (Weinberger \& Armsdorfer, 2004). In other words, the absorption coefficient of the far-infrared and the mean free path of the dust was of the same order. 

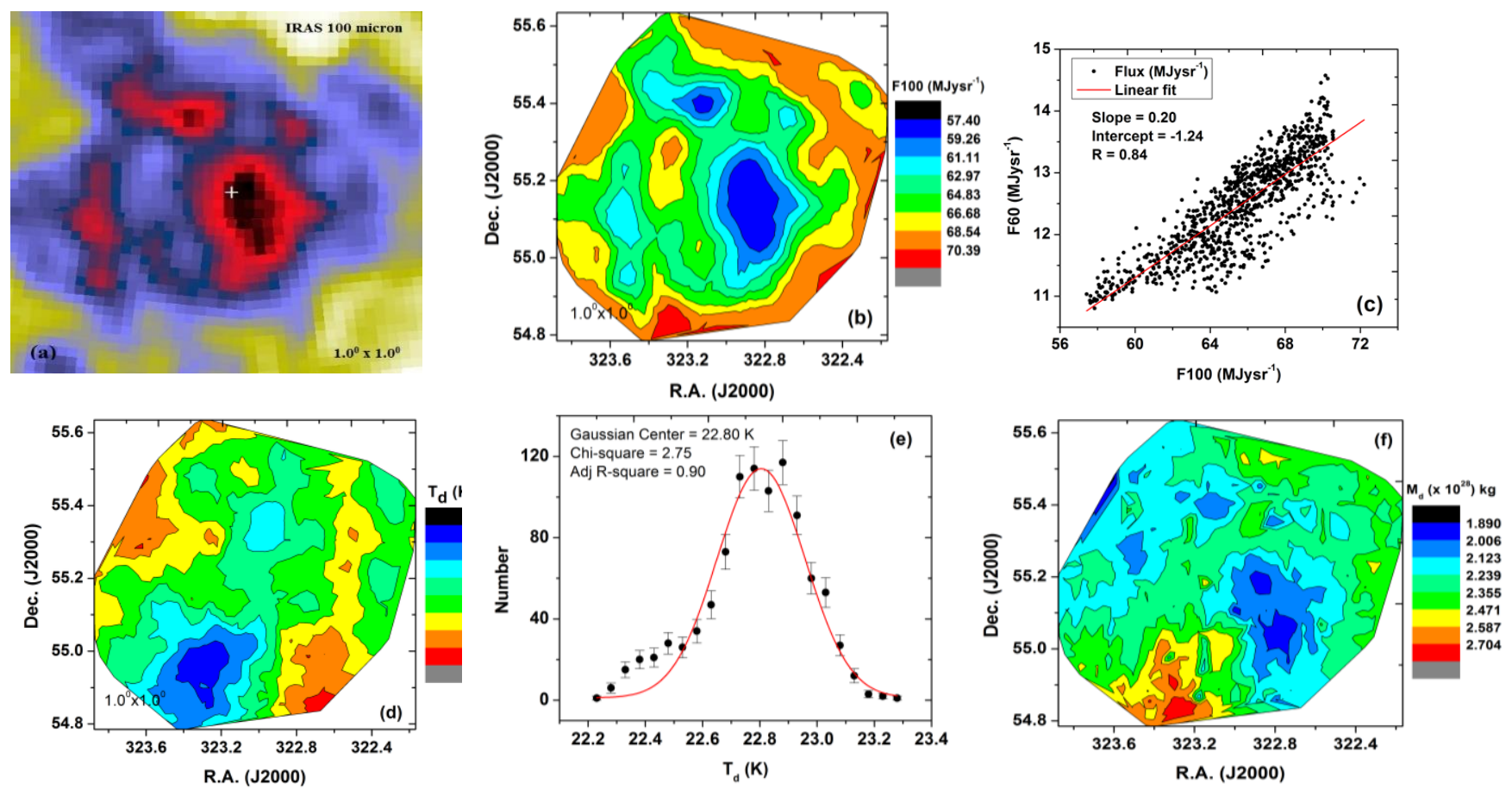
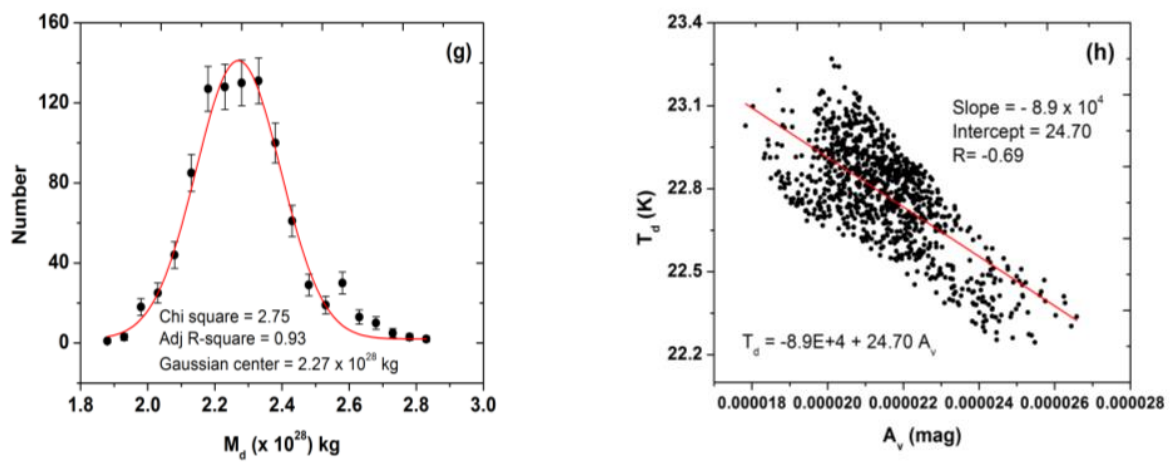

Figure 1. (a) JPEG image, (b) contour map of flux density, (c) linear fit of $100 \mu \mathrm{m}$ vs. $60 \mu \mathrm{m}$ flux density, (d) contour plot of dust temperature, (e) histograms of estimated temperature vs. number of image pixels, (f) contour map of dust mass, (g) histograms of estimated mass vs. number of image pixels, (h) linear fit between temperature and visual extinction, for the cavity structure FIC21+55.

From the comparison of the contour plot of temperature distribution (Figures 1(d) and 2(d)) with the distribution of flux density (Figures 1(b) and 2(b)), we observed that the minimum temperature region did not exactly coincide with the minimum-flux region for both studied cavities. Such a shifting trend was also observed in previous sources (e.g., Sapkota \& Aryal, 2019; Gautam et al., 2020; Tiwari et al., 2021); this unusual behavior can be expected due to the effect of nearby sources (e.g., AGB wind) (Gautam et al., 2020; Tiwari et al., 2021). Figures 1(e) and 2(e) represent histograms of the number of pixels with a temperature between $T_{d}$ to $T_{d}+\Delta\left(T_{d}\right)$ within the cavity structures FIC21+55 and FIC16-56, respectively. The dust color distribution of FIC21+55 showed a Gaussian nature with the value of Gaussian center $22.80 \mathrm{~K}$, whereas a positively skewed Gaussian-like nature with a Gaussian center 25.43
K was observed for FIC16-56. Dust mass of the structure was calculated using Equation 4, where the distance to the structure $(\mathrm{D})=\sim 3580 \mathrm{pc}($ for FIC21 +55$)$ and $2449 \mathrm{pc}$ for (FIC16-56) was used. The total mass of the cavity structure FIC21+55 was obtained to be $2.19 \times 10^{31} \mathrm{~kg}$ with an average mass of $2.28 \times 10^{28} \mathrm{~kg}$. On the other hand, a total mass of $1.59 \times 10^{30} \mathrm{~kg}$ with an average mass of $4.16 \times 10^{27} \mathrm{~kg}$ was estimated for the cavity FIC15-56. The contour map of dust mass of the cavity structures FIC21+55 and FIC1656 are shown in Figures 1(f) and 2(f), respectively. The contour plots of dust color temperature (Figures 1(d) and 2(d)) and the dust mass of the cavity (Figures 1(f) and 2(f)) showed that the majority of the minimum temperature region corresponds to a denser mass region. This phenomenon indicates a homogeneous and isotropic distribution of the dust within the structures (Gautam \& Aryal, 2020). 

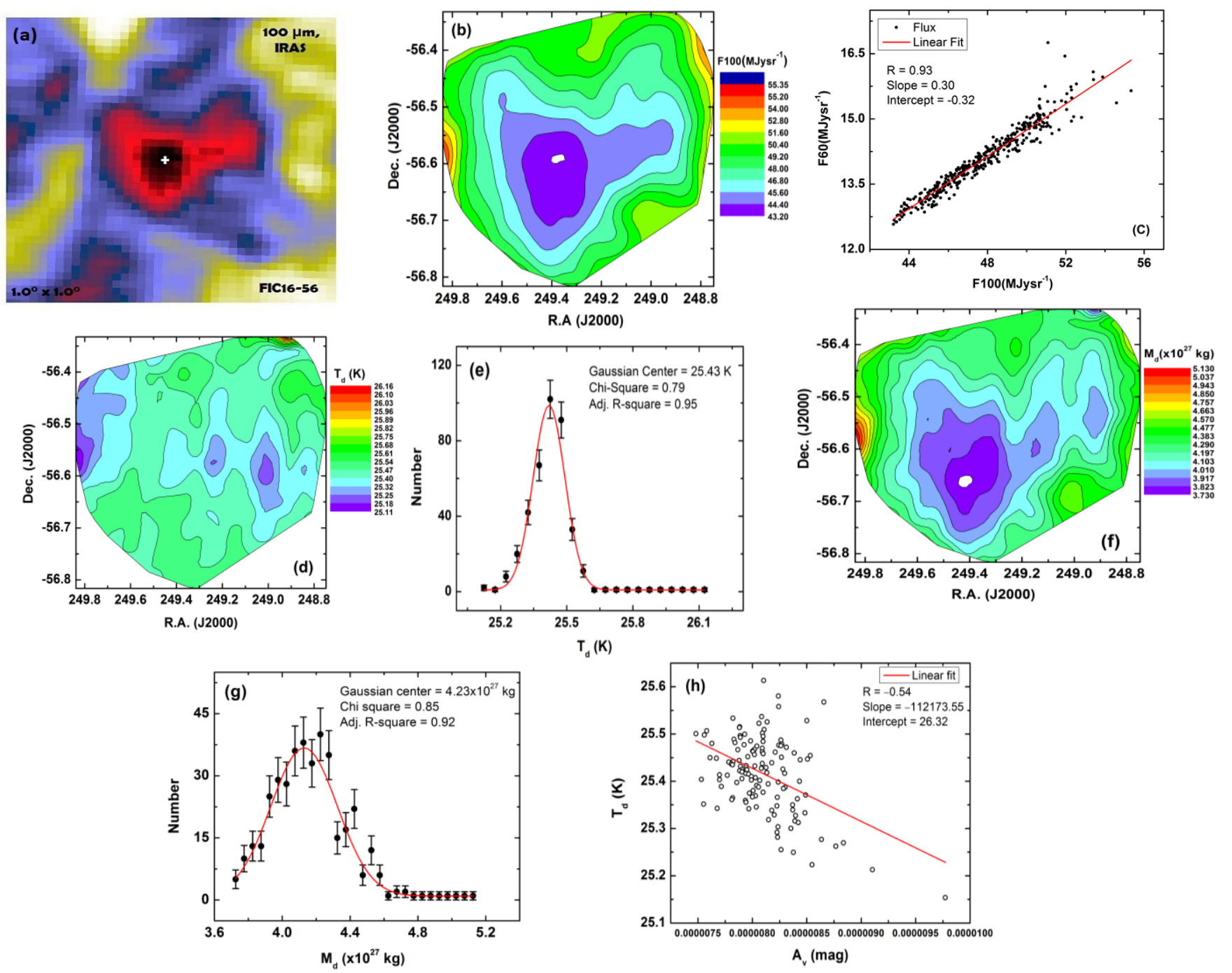

Figure 2. (a) JPEG image, (b) contour map of flux density, (c) linear fit of $100 \mu \mathrm{m}$ vs. $60 \mu \mathrm{m}$ flux density, (d) contour plot of dust temperature, (e) histograms of estimated temperature vs. number of image pixels, (f) contour map of dust mass, (g) histograms of estimated mass vs. number of image pixels, (h) linear fit between temperature and visual extinction, for the cavity structure FIC16-56.
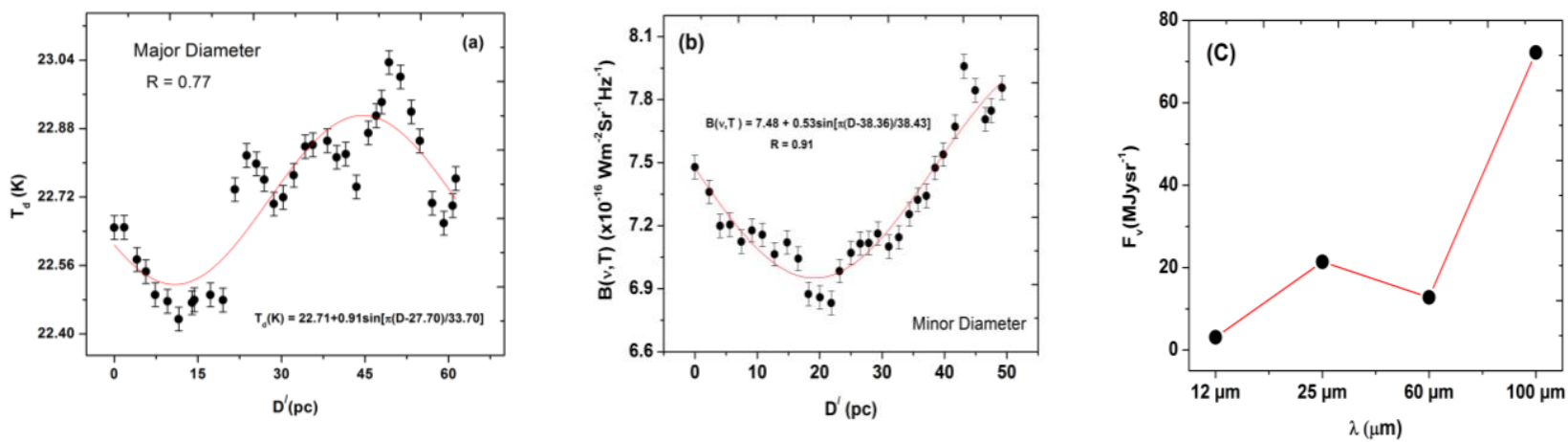

Figure 3. (a) Sinusoidal fit of Planck function over a distance along the major diameter, (b) sinusoidal fit of Planck function over a distance along the minor diameter, (c) far-infrared spectral distribution, of the cavity structure FIC21+55. 
Histograms of the number of pixels with a temperature between $M_{d}$ to $M_{d}+\Delta\left(M_{d}\right)$ within the cavities FIC21+55 and FIC16-56 is shown in Figures $1(\mathrm{~g})$ and $2(\mathrm{~g})$, respectively. The distribution of dust mass showed a Gaussian trend, indicating symmetric behavior of dust mass. Moreover, the unusual behavior of shifting of minimum temperature region and the maximum mass region in the cavity FIC16-56 was also supported by the Gaussian plots of dust temperature and mass (Figures 2(e) and $2(\mathrm{~g}))$. The value of optical depth was used in Equation (5) to calculate visual extinction at each pixel within the studied cavities. Figures $1(\mathrm{~h})$ and $2(\mathrm{~h})$ show the linear fit between visual extinction and dust color temperature; these plots showed the possible linear relationship between $\mathrm{Td}$ and Au with a negative correlation $(\mathrm{R})=-0.63$ for FIC21 +55 , and $\mathrm{R}=-0.54$ for FIC16-56. Since the value of Au was greater in a denser mass region, which implies a smaller value of temperature in the corresponding region. As the minimum temperature region was shifted from the maximum mass region (maybe due to possible external effects), it might have pushed to the lower value of the correlation coefficient between $\mathrm{Td}$ and Av. The product of $\mathrm{Td}$ and $\mathrm{Au}$ was less than one, i.e., $\mathrm{A}_{\mathrm{v}} \times \mathrm{T}_{\mathrm{d}}<1$, indicating that the lower visual extinction for the higher temperature and vice versa. These results were also in accordance with the previous findings (e.g., Gautam \& Aryal, 2019a, Gautam \& Aryal, 2019b); however, they have estimated a slightly larger value of correlation coefficients between the temperature of dust and visual extinction.
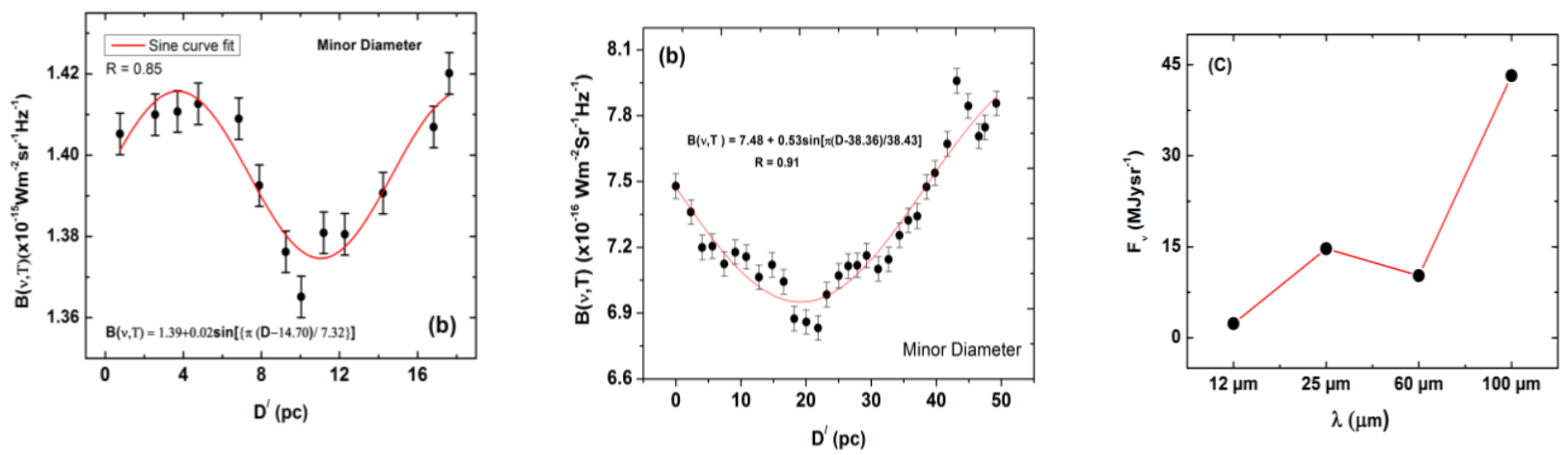

Figure 4. (a) Sinusoidal fit of Planck function over a distance along the major diameter, (b) sinusoidal fit of Planck function over a distance along the minor diameter, (c) far-infrared spectral distribution, of the cavity structure FIC16-56.

Figures 3(a), 3(b) and Figures 4(a), 4(b) show the plots of variation of Planck function along major and minor diameters passing through the flux minima at 100 microns of the cavity structures FIC21+55 and FIC16-56, respectively. These distributions followed the sinusoidal trend, indicating that the particles along both diameters of studied cavities were not in thermal equilibrium and oscillated to acquire dynamical equilibrium (Tiwari et al., 2021). This nature of oscillation was well-described in previous literature (Gautam \& Aryal, 2019a).

Figures 3(c) and 4(c) show the FIR spectral distribution of the cavity structures FIC21+55 and FIC16-56, respectively. In both structures, it has a positive slope in between the flux at the wavelengths 12 to 25 microns as well as 60 to 100 microns; however, a negative slope was noticed between the flux at wavelengths 60 to 100 microns, suggesting lower dust particles number density at 60micron band. Possibly, as a consequence of the interplay between the AGB wind and the ambient interstellar medium, the far-infrared cavity has a notable drop at 60micron flux density instead of a continuous rise. FIR spectral distribution with skeleton nebula (Aryal \& Weinberger, 2006) and high latitude molecular cloud (Weiland et al., 1986) showed continuous rise with different slopes. This discrepancy in the nature of FIR indicated the possible role of AGB wind. As the cavity emitted less radiation at a 60-micron wavelength and thus signified the deficiency of dust and grains in the cavities. These materials were expected to be swept away by the AGB wind from the cavity. This phenomenon was also observed in the case of AGBs and pulsars (Weiland et al., 1986; Ferriere, 2001; Suh \& Kwon, 2009; Gautam \& Aryal, 2019a).

Furthermore, we calculated the major and minor diameters of the selected far-infrared cavity using Equation (7). The value of distance $\left(D^{\prime}\right)=\sim 3580$ pc for FIC21 +55 and 2449 pc for FIC16-56 were used, and the pixel size (in radian) of the structure was measured using ALADIN v2.5. The distance of the major and minor axis of FIC21+55 was measured to be $\sim 61.8 \mathrm{pc}$ and $\sim 46.5 \mathrm{pc}$, and of FIC16-56 was estimated to be $\sim 25.55 \mathrm{pc}$ and $\sim 17.92 \mathrm{pc}$, respectively. Hence, the size of the FIC21+55 and FIC16-56 were estimated as $61.8 \mathrm{pc} \times 46.5 \mathrm{pc}$ and $25.55 \mathrm{pc} \times 17.92 \mathrm{pc}$, respectively.

\section{CONCLUSIONS}

This paper elucidated the physical properties such as dust color temperature, dust mass, Planck function, and visual extinction of the far-infrared cavity structures, namely FIC21 +55 and FIC16-56 at $60 \mu \mathrm{m}$ and $100 \mu \mathrm{m}$ IRAS maps located nearby AGB stars within $-10^{\circ}<\mathrm{b}<10^{\circ}$ Galactic planes. We summarized the results as follows: 
- The values of dust color temperature were found in the range $22.24 \pm 0.81 \mathrm{~K}$ to $23.27 \pm 0.21 \mathrm{~K}$ for $\mathrm{FIC} 21+55$, and $25.12 \pm 0.43 \mathrm{~K}$ to $26.17 \pm 0.62 \mathrm{~K}$ for FIC16-56, with an offset of about $1 \mathrm{~K}$.

- The size of the structures, FIC21+55, and FIC16-56, were found to be $61.8 \mathrm{pc} \times 46.5 \mathrm{pc}$ and $25.55 \mathrm{pc} \times$ $17.92 \mathrm{pc}$, respectively.

- The majority of the minimum flux regions corresponded to the minimum mass regions in both cavities. This suggests that the distribution of dust was homogeneous and isotropic. However, the minimum flux-region was shifted from the minimum temperature region of the cavity. Moreover, although the dust color temperature and dust mass distributions in the cavities showed the Gaussian (like) nature, for temperature vs. number plot of FIC $21+55$, we see some bumps at the beginning. These unusual behavior indicated the possible effect of nearby sources (e.g., AGB wind).

- The distribution of Planck function along both of the diameters in both structures was sinusoidal. This indicated that the particles along these diameters were oscillating.

- We observed a negative correlation between Au and $\mathrm{Td}$ in both cavities; less value of correlation coefficient between $\mathrm{Au}$ and $\mathrm{Td}$ was obtained in comparison to previous studies; we expected the role of external sources behind this.

- There was a significant decrease in the flux density between $25 \mu \mathrm{m}$ to $60 \mu \mathrm{m}$ wavelengths in FIR spectral distribution for both cavities. This significant dip at 60 $\mu \mathrm{m}$ instead of the continuous increase was possible due to the interaction of $A G B$ wind with the interstellar medium.

Hence, dust grains obscure the light from distant stars, track physical parameters (such as Galactic magnetic fields and gas temperature) and have direct interaction with other interstellar medium components (e.g., the formation of molecules and stars) (Williams, 2005). It also has a direct impact on the star formation processes. Due to this reason, the study of dust around different environments in the ISM is a crucial task. Dust properties trend nearby different AGB stars can be revealed by a model from an extensive number of similar works. Further, the rate of mass loss from the AGB star also can be studied. Following these methods, comparative study of dust properties of other large numbers of new cavity structures nearby various sources such as AGSs, Pulsars, White Dwarfs have to be carried out in the future. It provides crucial information for a better understanding of a dust grain status in the ISM. We believe this research work will contribute as a block of references for future studies about the shaping process of the Interstellar Medium.

\section{ACKNOWLEDGEMENTS}

We are thankful to Prof. Dr. Binil Aryal, Dean, Institute of Science and Technology (IoST), Tribhuvan University, for for his valuable suggestions. We extend our acknowledgements to Sky View virtual Observatory, IRAS Survey, SIMBAD database, Suh and Kwok catalog, Gaia DR2, ALADIN v2.5, and ORIGIN v8.5.

\section{AUTHOR CONTRIBUTIONS}

S.P. Gautam and A. Silwal developed the idea of this manuscript. They have performed all the computational works required in this paper. The authors M. Tiwari, S. Subedi, and M. Khanal have an equal combined effort to prepare the introduction, methodology, results, and conclusion section. This work was conducted under the guidance of the research advisor A.K. Jha.

\section{CONFLICT OF INTEREST}

The authors declare no conflict of interest.

\section{DATA AVAILABILITY STATEMENT}

The data that support the findings of this study are available from the corresponding author, upon reasonable request.

\section{REFERENCES}

Arce, H.G., \& Goodman, A.A. (1999). An extinction study of the Taurus dark cloud complex. Astrophysical Journal, 517, 264-281. Aryal, B., \& Weinberger, R. (2006). A new large high latitude conelike far-IR nebula. Astronomy \& Astrophysics, 448(1), 213-219.

Aryal, B., Rajbahak, C., \& Weinberger, R. (2010). A giant dusty bipolar structure around the planetary nebula ngc 1514. Monthly Notices of the Royal Astronomical Society, 402, 1307-1312.

Beichman, C.A., Wilson, R.W., Langer, W.D., \& Goldsmith, P.F. (1988). Infrared limb brightening in the Barnard 5 cloud. The Astrophysical Journal, 332, L81-L85.

Bowen, G.H. (1988). Dynamical modeling of long-period variable star atmospheres. The Astrophysical Journal, 329, 299-317.

Cernicharo, J., Guélin, M., Agúndez, M., McCarthy, M.C., \& Thaddeus, P. (2008). Detection of C5N- and vibrationally excited C6H in IRC+ 10216. The Astrophysical Journal Letters, 688(2), L83.

Danchi, W.C., Bester, M., Degiacomi, C.G., Greenhill, L.J., \& Townes, C.H. (1994). Characteristics of dust shells around 13 late-type stars. The Astronomical Journal, 107, 1469-1513.

Dupac, X., Bernard, J.P., Boudet, N., Giard, M., Lamarre, J.M., Meny, C., Pajot, F., Ristorcelli, I., Serra, G., Stepnik, B., \& Torre, J.P. (2003). Inverse temperature dependence of the dust submillimeter spectral index. Astronomy \& Astrophysics, 404, L11-L15.

Ferriere, K.M. (2001). The interstellar environment of our galaxy. Reviews of Modern Physics, 73(4), 1031.

Gautam, A.K. \& Aryal, B. (2019a). A study of four low-latitude $\left(|1|<10^{\circ}\right)$ far-infrared cavities. Journal of Astronomy and Astrophysics, 40, 16.

Gautam, A.K. \& Aryal, B. (2019b). Study of two far-infrared cavities nearby asymptotic giant branch stars under infrared astronomical satellite maps. Journal of Institute of Science and Technology, 24(2), 76-84.

Gautam, A.K. \& Aryal, B. (2020). Study of dust color temperature and visual extinction distribution of a far-infrared cavity at 60 
and $100 \mu \mathrm{m}$ IRAS map around the AGB star at galactic latitude 8.6 $0^{\circ}$. BIBECHANA, 17, 42-49.

Gautam, S.P., Silwal, A., Jha, A.K. (2020). Dust color temperature and Planck function distribution of a far infrared planetary nebula at 90 and $140 \mu \mathrm{m}$ AKARI map. International Astronomy and Astrophysics Reserach Journal, 2(2), 1-8.

Gehrz, R.D. (1989). Sources of stardust in the galaxy. SymposiumInternational astronomical union, 135, Cambridge University Press, pp. 445-453.

Gobrecht, D., Cherchneff, I., Sarangi, A., Plane, J.M.C., \& Bromley, S.T. (2016). Dust formation in the oxygen-rich AGB star IK Tauri. Astronomy \& Astrophysics, 585, 15pp.

Groom, H. (2018). Dust formation and evolution around carbon stars. $\mathrm{PhD}$ Thesis. University of Missouri-Columbia.

Herwig, F. (2005). Evolution of asymptotic giant branch stars. Annual Review of Astronomy and Astrophysics, 43, 435-479.

Hildebrand, R.H., Whitcomb, S.E., Winston, R., Stiening, R.F., Harper, D.A., \& Moseley, S.H. (1977). Submillimeter photometry of extragalactic objects. The Astrophysical Journal, 216, 698-705.

Hildebrand, R.H. (1983). The determination of cloud masses and dust characteristics from submillimetre thermal emission. Quarterly Journal of the Royal Astronomical Society, 24, 267-282.

Iben Jr, I. (1967). Stellar evolution. VI. Evolution from the main sequence to the red-giant branch for stars of mass $1 \mathrm{M}_{-}\{\operatorname{sun}\}$, 1.25 M_\{sun\}, and 1.5 M_\{sun\}. The Astrophysical Journal, 147, 624-649.

Jha, A.K., \& Aryal, B. (2017). A study of pulsar wind driven structure in far-infrared IRAS map at latitude $-10^{\circ}$. Journal of Institute of Science and Technology, 22 (1), 1-9.

Jha, A. K., Aryal, B., \& Weinberger, R. (2017). A study of dust colour temperature and dust mass distributions of four farinfrared loops. Revista Mexicana de Astronomia y Astrofisica, 53, 467-476.

Jha, A.K., \& Aryal, B. (2018). Dust color temperature distribution of two FIR cavities at IRIS and AKARI maps. Journal of Astronomy and Astrophysics, 39(2), 7.

Justtanont, K., Khouri, T., Maercker, M., Alcolea, J., Decin, L., Olofsson, H., Schöier, F.L., Bujarrabal, V., Marston, A.P., Teyssier, D., Cernicharo, J., Dominik, C., de Koter, A., Melnick, G., Menten, K.M., Neufeld, D., Planesas, P., Schmidt, M., Szczerba, R., \& Waters, R. (2012). Herschel/HIFI observations of O-rich AGB stars: molecular inventory. Astronomy \& Astrophysics, 537, A144.

Kiss, C., Moór, A., \& Tóth, L.V. (2004). Far-infrared loops in the 2nd Galactic Quadrant. Astronomy \& Astrophysics, 418(1), 131141.

Könyves, V., Kiss, C., Moór, A., Kiss, Z.T., \& Tóth, L.V. (2007). Catalogue of far-infrared loops in the Galaxy. Astronomy \& Astrophysics, 463(3), 1227-1234.
Neufeld, D.A., González-Alfonso, E., Melnick, G., Szczerba, R., Schmidt, M., Decin, L., Alcolea, J., de Koter, A., Schöier, F.L., Bujarrabal, V., Cernicharo, J., Dominik, C., Justtanont, K., Marston, A.P., Menten, K., Olofsson, H., Planesas, P., Teyssier, D., \& Waters, L.B.F.M. (2011). The widespread occurrence of water vapor in the circumstellar envelopes of carbon-rich asymptotic giant branch stars: first results from a survey with HERSCHEL*/HIFI. The Astrophysical Journal Letters, 727(2), L29.

Rees, M.J. (1984). Black hole models for active galactic nuclei. Annual Review of Astronomy and Astrophysics, 22(1), 471-506.

Sapkota, B.B., \& Aryal, B. (2019). A study of far infrared cavity At-3.6 Galactic latitude. Journal of Nepal Physical Society, 5(1), 54-58.

Schnee, S.L., Ridge, N.A., Goodman, A.A., \& Li, J.G. (2005). A complete look at the use of IRAS emission maps to estimate extinction and dust temperature. The Astrophysical Journal, 634(1), 442.

Suh, K.W., \& Kwon, Y.J. (2009). A catalogue of AGB stars in IRAS PSC. Journal of the Korean Astronomical Society, 42, 81-91.

Tiwari, M., Gautam, S.P., Silwal, A., Subedi, S., Paudel, A., \& Jha, A.K. (2021). Study of dust properties of two far infrared cavities nearby asymptotic giant branch stars under Infrared Astronomical Satellite maps. Himalayan Physics, 9, 73-84.

Vassiliadis, E., \& Wood, P.R. (1993). Evolution of low-and intermediate-mass stars to the end of the asymptotic giant branch with mass loss. The Astrophysical Journal, 413, 641-657.

Ventura, P., Griscienzo, M., Schnieider, R., Carini, R., Valiante, R., D'Antona, F., Gallerani, S., Maiolino, R., \& Rornambé, A. (2012). Dust formation around agb and sagb stars: a trend with metallicity? Monthly Notices of Royal Astronomical Society, 424 (3), 2345-2357.

Weiland, J.L., Blitz, L., Dwek, E., Hauser, M.G., Magnani, L., \& Rickard, L.J. (1986). Infrared cirrus and high-latitude molecular clouds. The Astrophysical Journal, 306, L101-L104.

Weinberger, R., \& Armsdorfer, B. (2004). A pair of gigantic bipolar dust jets close to the solar system. Astronomy \& Astrophysics, 416(3), L27-L30.

Willacy, K., \& Cherchneff, I. (1998). Silicon and Sulphur chemistry in the inner wind of IRC+ 10216. Astronomy and Astrophysics, 330, 676-684.

Williams, D.A. (2005). Gas and dust in the interstellar medium. Journal of Physics: Conference Series, 6, 1-17.

Wood, D.O.S., Myers, P.C., \& Daugherty, D.A. (1994). IRAS images of nearby dark clouds. The Astrophysical Journal Supplement, 95, 457-501

Young, K., Phillips, T.G., \& Knapp, G.R. (1993). Circumstellar shells resolved in IRAS survey data. II-Analysis. The Astrophysical Journal, 409, 725-738. 